Prepublished online April 17, 2013;

doi:10.1182/blood-2012-12-471698

\title{
Immunogenetics shows that not all MBL are equal: the larger the clone the more similar to CLL
}

Anna Vardi, Antonis Dagklis, Lydia Scarfò, Diane Jelinek, Darren Newton, Fiona Bennett, Julia Almeida, Arancha Rodriguez-Caballero, Sallie Allgood, Mark Lanasa, Agostino Cortelezzi, Ester Orlandi, Silvio Veronese, Marco Montillo, Andy Rawstron, Tait Shanafelt, Alberto Orfao, Kostas Stamatopoulos and Paolo Ghia

Information about reproducing this article in parts or in its entirety may be found online at: http://bloodjournal.hematologylibrary.org/site/misc/rights.xhtml\#repub_requests

Information about ordering reprints may be found online at:

http://bloodjournal.hematologylibrary.org/site/misc/rights.xhtml\#reprints

Information about subscriptions and ASH membership may be found online at:

http://bloodjournal.hematologylibrary.org/site/subscriptions/index.xhtml

Advance online articles have been peer reviewed and accepted for publication but have not yet appeared in the paper journal (edited, typeset versions may be posted when available prior to final publication). Advance online articles are citable and establish publication priority; they are indexed by PubMed from initial publication. Citations to Advance online articles must include the digital object identifier (DOls) and date of initial publication.

Blood (print ISSN 0006-4971, online ISSN 1528-0020), is published weekly by the American Society of Hematology, 2021 L St, NW, Suite 900, Washington DC 20036.

Copyright 2011 by The American Society of Hematology; all rights reserved.

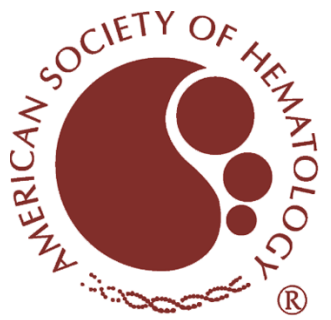




\section{IMMUNOGENETICS SHOWS THAT NOT ALL MBL ARE EQUAL: THE LARGER THE CLONE THE MORE SIMILAR TO CLL}

Running title: IG genes in MBL versus CLL

Anna Vardi ${ }^{1}$, Antonis Dagklis ${ }^{2}$, Lydia Scarfò ${ }^{2}$, Diane Jelinek ${ }^{3}$, Darren Newton ${ }^{4}$, Fiona Bennett $^{4}$, Julia Almeida ${ }^{5}$, Arancha Rodriguez-Caballero ${ }^{5}$, Sallie Allgood ${ }^{6}$, Mark Lanasa $^{6}$, Agostino Cortelezzi ${ }^{7,8}$, Ester Orlandi ${ }^{8,9}$, Silvio Veronese ${ }^{10}$, Marco Montillo $^{8,10}$, Andy Rawstron ${ }^{4}$, Tait Shanafelt ${ }^{3}$, Alberto Orfao ${ }^{5}$, Kostas Stamatopoulos ${ }^{1,11}$, Paolo Ghia ${ }^{2,8}$

1. Hematology Department and HCT Unit, G. Papanicolaou Hospital, Thessaloniki, Greece

2. Division of Molecular Oncology and Department of Onco-Hematology, Universita Vita-Salute San Raffaele and Istituto Scientifico San Raffaele, Milano, Italy

3. Department of Immunology, Mayo Clinic, Rochester, MN

4. Haematological Malignancy Diagnostic Service, St. James's Institute of Oncology, Leeds Teaching Hospitals, Leeds, United Kingdom

5. Centro de Investigación del Cáncer (CIC, IBMCC USAL-CSIC) and Department of Medicine, University of Salamanca, Salamanca, Spain

6. Department of Medicine, Duke University Medical Center, Durham, NC

7. Department of Medical Sciences, Hematology 1 CTMO, University of Milan, Fondazione IRCCS Ca' Granda Ospedale Maggiore Policlinico, Milan, Italy

8. Rete Ematologica Lombarda (REL), Italy

9. Department of Hematology Oncology, Fondazione IRCCS Policlinico San Matteo \& University of Pavia, Pavia, Italy

10. Niguarda Ca' Granda Hospital, Milan, Italy

11. Institute of Applied Biosciences, Center for Research and Technology Hellas, Thessaloniki, Greece 


\section{Corresponding author:}

Kostas Stamatopoulos, MD, PhD

Hematology Department and HCT Unit

G. Papanicolaou Hospital

57010 Exokhi, Thessaloniki, GREECE

Phone: $+30.2313 .307992 /$ Fax: +30.2313 .307579$

e-mail: kostas.stamatopoulos@gmail.com 


\section{KEY POINTS}

- Low count and high count monoclonal B lymphocytosis (MBL) have distinct immunogenetic signatures, with only the latter resembling CLL.

- Rather than a true premalignant condition, low count MBL may merely reflect immune senescence or result from persistent antigen stimulation.

\section{ABSTRACT}

CLL-like monoclonal B lymphocytosis (MBL) shares common immunophenotype and cytogenetic abnormalities with CLL, from which it is discriminated by a cut-off value of $5 \times 10^{9} / /$ circulating clonal $\mathrm{B}$ cells. However, the clonal size amongst $\mathrm{MBL}$ is extremely variable and allows discrimination of two distinct entities (High- and Lowcount MBL) based on a cut-off value of $0.5 \times 10^{9} / /$ clonal B cells. High-count (HC) MBL is associated with lymphocytosis and progresses to CLL requiring treatment at a rate of $1.1 \%$ /year, whereas low-count (LC) MBL is found in the general population only through high-sensitivity techniques and carries limited -if any- risk of progression. We performed an immunogenetic profiling of 333 CLL-like MBL cases supplemented by detailed comparisons with CLL, focusing especially on CLL Rai stage-0 (CLL-0). LC and HC-MBL had similar somatic hypermutation status, yet different IGHV gene repertoires and frequencies of $B$-cell receptor $(B c R)$ stereotypy. In particular, stereotyped BcRs were infrequent in LC-MBL and often not CLL-specific. In contrast, HC-MBL exhibited clear immunogenetic similarities to CLL-0, extending to the frequent presence of CLL-specific stereotypes. These findings indicate that LC-MBL may not represent a true preleukemic condition, thus differing from HC-MBL/CLL-0 where the identification of factors endowing malignant potential is strongly warranted. 


\section{INTRODUCTION}

The existence of monoclonal B cell expansions in the peripheral blood (PB) of healthy individuals has long been known, ${ }^{1-3}$ but has recently been more clearly appreciated and dissected thanks to advanced flow cytometry techniques. ${ }^{4-7}$ This condition falls under the general term "monoclonal B cell lymphocytosis" (MBL), even though it may not always correspond to an increase in the absolute lymphocyte count. MBL is classified according to the immunophenotype of the clonal population. Thus, the majority of $\mathrm{MBL}$ cases (75\%) have the immunophenotype of chronic lymphocytic leukemia $\left(\mathrm{CD}^{+}, \mathrm{CD} 23^{+}, \mathrm{CD} 20^{\mathrm{dim}}\right.$, slg $\left.{ }^{\mathrm{dim}}\right)$ and are defined as CLL-like $\mathrm{MBL}$, whereas the remaining MBL cases are classified as atypical CLL-like (CD5 ${ }^{+}$, $\mathrm{CD}^{\text {bright }}$ ) and CD5-negative (CD5 ${ }^{-} \mathrm{MBL}^{8,9}$

CLL-like MBL is reported to be a premalignant state of CLL, analogous to the relationship between monoclonal gammopathy of undetermined significance and multiple myeloma. ${ }^{10}$ Its frequency ranges between 3 and $12 \%$, depending on the sensitivity of the techniques utilized, and reaches $17 \%$ among CLL family members. ${ }^{5,6,11}$

The World Health Organization (WHO) has set a consensus cut-off value of $5 \times 10^{9} / \mathrm{I}$ clonal B cells in the PB to discriminate what constitutes "disease" and what not. ${ }^{12}$ That notwithstanding, below this limit the size of the clone both in absolute numbers as well as in percentage of circulating $B$ cells is very heterogeneous, and MBL is now usually subdivided into two categories: "High-count" MBL (HC-MBL, also known as clinical MBL) and "Low-count" MBL (LC-MBL, also called population-screening MBL). ${ }^{8,13}$ The former is usually associated with lymphocytosis and is recognized in a clinical setting, thus explaining the initial name. ${ }^{14}$ The HC-MBL cases may progress to CLL requiring treatment at a rate of $1-2 \%$ per year. ${ }^{14}$ On the other hand, LC-MBL can only be detected by applying highly sensitive cytometric techniques in otherwise healthy individuals ${ }^{13}$, and has been described to remain stable over time, with no case progressing into HC-MBL or CLL within a 34-month follow-up time. ${ }^{15}$

The jury is still out as to what, if any, is the best cut-off value for discriminating MBL into sub-entities with truly distinct biological and clinical significance. In clinical MBL, the clonal size in the PB has a median value of about $2.9 \times 10^{9} \mathrm{~B}$ cells/l and $95 \%$ of cases have more than $0.45 \times 10^{9}$ clonal $B$ cells $/{ }^{16}{ }^{16}$ whereas in LC-MBL the median number of clonal $\mathrm{B}$ cells is only $0.001 \times 10^{9} / \mathrm{I}^{13}$ For all these reasons, a cut-off value of $0.5 \times 10^{9}$ clonal B cells/l has been proposed for discriminating LC versus HC-MBL, based on the actual risk of progression. ${ }^{8,13}$ 
Thus, overall, the term MBL comprises a mixture of entities with different likelihood of representing a true preleukemic condition, as also indicated by the clinical data., ${ }^{4,15}$ With this in mind, in the present paper we further pursue the possibility to understand the relationship between the different $\mathrm{MBL}$ cases and CLL by analyzing the immunoglobulin gene repertoire within a cohort of 333 individuals with CLL-like MBL, both LC ( $n=60)$ and HC ( $n=273)$, from collaborating institutions in Europe and the U.S. Comparisons of LC-MBL and HC-MBL to a large cohort of CLL cases and to a subgroup of Rai stage 0 CLL cases (CLL-0) revealed that LC and HC-MBL have different immunogenetic profiles and in particular that HC-MBL resembles CLL-0, whereas LC-MBL is clearly distinct from CLL. On these grounds, we believe that a reappraisal of $\mathrm{MBL}$ and its relationship to CLL is strongly warranted and clinically relevant. 


\section{PATIENTS AND METHODS}

\section{Patient group}

A total of 333 patients diagnosed with CLL-like MBL from collaborating institutions in Europe and the US were included in the present study. All cases met the formal criteria for the diagnosis of MBL established in $2005 .{ }^{9}$ In particular, CLL-like MBL was defined on the basis of an unbalanced $k / \lambda$ ratio $(>3: 1$ or $<1: 3)$ within $C D 19^{+}, C^{\text {bright }}$, $\mathrm{CD}^{2} 3^{+}, \mathrm{CD} 20^{\text {dim }}$ cells determined by flow cytometry. Within this cohort, 60 cases had a clonal B cell count of less than $0.5 \times 10^{9} /$ ("low count", LC-MBL), whereas the remaining 273 cases had from 1.5 to less than $5.0 \times 10^{9} / /$ clonal $B$ cells with a CLLlike phenotype ("high count", HC-MBL) (Table 1). There was no case of LC-MBL with increased $\mathrm{T}$ cells or polyclonal $\mathrm{B}$ cells that would result in absolute lymphocytosis. The distribution of MBL cases according to clonal size is depicted in Figure 1. The study was approved by the local Ethics Review Committee of each institution. The study was conducted in accordance with the Declaration of Helsinki.

\section{PCR amplification of IGHV-IGHD-IGHJ rearrangements}

PCR amplification of IGHV-IGHD-IGHJ rearrangements were performed on either genomic DNA (gDNA) or complementary DNA (cDNA), as previously described. ${ }^{4,17}$

\section{Sequence analysis and interpretation}

PCR amplicons were subjected to either direct sequencing on both strands or, in 15 cases, after subcloning; in the latter case, at least 10 colonies were analyzed per amplicon.

Sequence data were analyzed using the IMGT® databases $^{18}$ and the IMGT/VQUEST tool (http://www.imgt.org). ${ }^{19}$ Codons and amino acid positions are according to the IMGT unique numbering for $V$ domain. ${ }^{20}$ Only productive rearrangements were evaluated $(n=355)$.

Rearrangements utilizing the IGHV4-59 and IGHV4-61 genes were considered as one group because the usage of an FR2-primer for certain PCR amplification experiments precluded a clear assignment to either one or the other IGHV gene that differ mainly in the VH FR1-VH CDR1. In order to avoid misidentification of mutations when IGHV FR1 or FR2 consensus primers were used in the amplification reactions, nucleotide substitutions in the obtained sequences were evaluated from codon 27 in CDR1-IMGT or from codon 56 in CDR2-IMGT in those sequences obtained with a VH FR2 primer. ${ }^{4}$ 


\section{IG repertoire comparisons and VH CDR3 clustering}

IG gene repertoire comparisons were performed for the IGHV-IGHD-IGHJ sequences from MBL cases included in the present study and 7596 IGHV-IGHDIGHJ sequences from a cohort of $7424 \mathrm{CLL}$ cases from our recent publication. ${ }^{21}$ Comparisons were also performed between MBL and a subgroup of the CLL cohort including only Rai stage 0 cases from three collaborating institutions within our network (San Raffaele, Milan; G. Papanicolaou, Thessaloniki; Nikea General Hospital, Athens) attending patients with CLL found in everyday clinical practice, i.e. without any selection bias ("community CLL") ( $\mathrm{n}=544$ cases).

Furthermore, MBL sequences from our series were aligned and compared to a panel of 5494 non-redundant, well annotated and productive IGHV-IGHD-IGHJ sequences from various entities except CLL which were retrieved from the IMGT/LIGM-DB sequence database (http://www.imgt.org/IMGTindex/LIGM.html); this panel has also been used for the same purpose in previous repertoire studies from our group., ${ }^{4,22,23}$ In order to assess BcR IG stereotypy within the entire collection of IGHV-IGHD-IGHJ sequences (MBL, CLL, other entities) we followed a clustering process based on purpose-built bioinformatics as previously described. ${ }^{22}$

\section{Statistical analysis}

Descriptive statistics for discrete parameters included counts and frequency distributions. For quantitative variables, statistical measures included means, medians, and min-max values. Significance of bivariate/multivariate relationships between variables was assessed with the use of chi-square. For all comparisons, a significance level of $p=0.05$ was set and all statistical analyses were performed with the statistical package GraphPad Prism version 5.0 (GraphPad Software, Inc., San Diego, USA). 


\section{RESULTS}

\section{Low-count and high-count MBL have distinct IG gene repertoires}

Overall, 355 productive IGHV-IGHD-IGHJ rearrangements were amplified from 333 cases with CLL-like MBL. When we differentiated MBL based on the absolute clonal B cell count - LC-MBL, $<0.5 \times 10^{9} / /$ (median $0.008 \times 10^{9} / /$ ); HC-MBL, $\geq 0.5 \times 10^{9}$, $<5.0 \times 10^{9} / \mathrm{l}$ (median $3.38 \times 10^{9} / \mathrm{l}$ ) (Table 1 ) - we noted that a significantly higher proportion of LC-MBL versus HC-MBL carried multiple productive IGHV-IGHD-IGHJ rearrangements $(7 / 60(11.7 \%)$ versus $8 / 273(2.9 \%)$ cases, $p=0.003)$. Among all studied rearrangements, IGHV3 was the predominant subgroup (175/355, 49.3\%), followed by IGHV4 (87/355, 24.5\%). Thirty-nine functional IGHV genes were identified. The most frequent gene was IGHV4-34 (42 rearrangements, 11.8\%) followed by IGHV3-23 (8.7\%), IGHV3-7 (8.5\%), IGHV1-69 (6.8\%) and IGHV4-59/61 (6.8\%) (Supplemental Table 1).

Significant differences were identified regarding the frequencies of IGHV genes between LC-MBL versus HC-MBL. At subgroup level, IGHV4 genes were significantly $(p=0.02)$ under-represented and IGHV1 genes were significantly overrepresented $(p=0.04)$ in HC-MBL vs. LC-MBL. At the individual gene level, the IGHV1-69, IGHV2-5, IGHV3-23, IGHV3-33, IGHV3-48 and IGHV4-34 genes predominated in HC-MBL vs. LC-MBL, however the observed differences did not reach statistical significance likely due to small sample size (at least for statistical purposes). With this in mind (i.e. relatively low numbers), it is indeed remarkable that the IGHV4-59/61 gene was highly significantly $(\mathrm{p}<0.0001)$ more frequent in LC-MBL versus HC-MBL (Figure 2, Supplemental Table 1).

Regarding VH CDR3 length and composition as well as IGHD and IGHJ gene usage, detailed results are provided in Supplemental Tables 2-4.

Following the 98\% germline identity (Gl) cut-off value, 267/355 (75.2\%) rearrangements were assigned to the mutated subgroup, whereas the remaining $(88 / 355,24.8 \%)$ were classified as unmutated, of which 52/88 (59.1\%) had $100 \%$ sequence identity to the corresponding germline IGHV gene. No differences were identified in terms of somatic mutations in LC vs. HC-MBL as evidenced by an overall similar distribution of cases to the mutated vs. unmutated subgroups. Interestingly, however, in MBL cases with multiple productive IGHV-IGHD-IGHJ rearrangements, the mutational status was generally concordant in LC-MBL (5/7 cases, $71.5 \%$, with only mutated IGHV genes) whereas it was often discordant in HC-MBL (5/8 cases, $62.5 \%$, with co-existing mutated and unmutated IGHV genes). 
Low-count MBL have a distinct IG gene repertoire whereas high-count MBL resemble Rai stage 0 CLL

We next compared the IG gene repertoire between MBL and CLL. To this end, we used as reference two CLL datasets, one exclusively comprising 544 CLL-0 cases and the other one 7424 cases in all clinical stages (thereafter designated as CLL-all stages, as detailed in the Patients and Methods section). In keeping with our previous report, ${ }^{4}$ the most pronounced difference concerned the IGHV4-59/61 gene, which was significantly over-represented in LC-MBL $(p<0.0001)$ (Figure 2). A significantly different distribution was also noted for the IGHV1-69 gene, which was rare in LC-MBL (2.8\%), utilized with similar, higher frequencies in HC-MBL and CLL0 (7.8\% and $5.5 \%$, respectively) and peaked at $12.8 \%$ in CLL-all stages, being the most frequent gene overall $(p<0.0001)$. Other examples of IGHV genes with asymmetric usage in the compared groups are given in Supplemental Table 5.

Regarding the mutational status of the IG rearrangements, no differences were identified between LC-MBL versus HC-MBL versus CLL-0, as the frequency of unmutated rearrangements ( $\geq 98 \% \mathrm{Gl}$ ) was overall similar (LC-MBL: $26.4 \%$, HC-MBL: 24.4\%, CLL-0: 25\%). Hence, all the aforementioned subgroups differed significantly $(p=0.001, p<0.0001$, and $p<0.0001$, respectively) from CLL-all stages where almost 1 out of $2(45.1 \%)$ rearrangements carried unmutated IGHV genes (Figure 3). Interestingly, in both HC-MBL and CLL-0, a sizeable proportion, roughly one-third of rearrangements utilizing the IGHV1-69 gene were mutated $(7 / 22,31.8 \%$ and $9 / 30$, $30 \%$, respectively), thus differing significantly $(p<0.001)$ from CLL-all stages, where less than $10 \%$ of all such rearrangements were mutated. In the LC-MBL group, only 2 cases utilized the IGHV1-69 gene, and it was mutated in both.

\section{$B c R$ stereotypy is infrequent in low-count $M B L$, with the occasional stereotyped BcRs constituting "public" immunogenetic signatures}

Analysis of the VH CDR3 region was possible in 349/355 rearrangements of the MBL cohort. In order to identify stereotyped VH CDR3s, we applied our purpose-built algorithm for the identification of shared VH CDR3 amino acid patterns and performed cluster analysis of the MBL sequences. ${ }^{22}$ Next, we also compared the MBL VH CDR3 dataset against 7596 CLL sequences from our recent publication. ${ }^{21}$ Finally, we also performed sequence comparisons with 5494 non-redundant, wellannotated and complete $\mathrm{VH}$ CDR3 sequences from various entities except CLL which were retrieved from the IMGT/LIGM-DB sequence database.

When we compared the frequency of "CLL-specific" BcR stereotypes between LC$M B L$ and HC-MBL, this was significantly higher in HC-MBL $(p=0.001)$, further 
underscoring a difference in the immunogenetic profile between these two entities. Specifically, only 4/72 (5.5\%) LC-MBL IG sequences, none from cases with multiple productive IGHV-IGHD-IGHJ gene rearrangements, could be clustered with other sequences in subsets with stereotyped BcRs; they concerned cases with clonal $B$ cells in the range of $0.004-0.055 \times 10^{9} /$. In particular, (i) 1 LC-MBL case was clustered together with a HC-MBL case, but with no CLL cases, thus forming a novel "MBL-specific" subset; (ii) 1 LC-MBL case could be assigned to a "CLL-specific" subset (subset \#239); (iii) 2 LC-MBL cases were clustered together with sequences from various entities, including CLL, thus they were considered to carry "public" BcR stereotypes (see following section).

In contrast, 62/283 (21.9\%) HC-MBL rearrangements carried VH CDR3s similar to either other MBL or CLL cases; $3 / 62$ were detected in cases with multiple rearrangements. In particular, (i) $45 \mathrm{HC}-\mathrm{MBL}$ cases clearly belonged to wellestablished "CLL-specific" subsets (e.g. \#1, \#2, \#4, \#16, \#28 etc); (ii) 10 HC-MBL cases clustered together with CLL, forming novel "MBL-CLL" subsets; (iii) 5 HC-MBL cases clustered together, forming "MBL-specific" subsets; and, (iv) $2 \mathrm{HC}-\mathrm{MBL}$ cases carried "public" IG BcR stereotypes, i.e. were assigned to clusters including sequences from various entities (see following section).

Furthermore, a progressive increase in the frequency of $\mathrm{BcR}$ IG stereotypy depending on the absolute count of CLL-like cells could be observed, starting with $5.5 \%$ in LC-MBL, rising to $21.9 \% / 20.2 \%$ in HC-MBL/CLL-0, respectively, and peaking at $30.4 \%$ in CLL-all stages (Figure 4).

\section{Public BcR IG stereotypes in the MBL repertoire}

The first LC-MBL case with a "public" BcR IG stereotype (89VBB) was assigned to a subset of sequences from mainly CLL (subset \#13) though also including other sequences as well (a rheumatoid factor from a healthy individual, the clonotypic BcR of a case with splenic marginal-zone lymphoma, two cases with Sjögren's syndromeassociated myoepithelial sialadenitis (SS-MESA) and a CLL case with prior HCVassociated type-II mixed cryoglobulinemia (HCV/MC-II). As we recently reported, ${ }^{24} \mathrm{a}$ number of cases assigned to this subset, regardless of the underlying diagnosis, had positive HCV serology; this was the case for 89VBB as well.

The second LC-MBL case with a "public" BcR IG stereotype (87VB) carried a VH CDR3 similar to the one recently reported in our study of ocular adnexa marginalzone lymphoma (OAMZL) (Figure 5A). ${ }^{25}$ This particular stereotype has so far been detected among various entities in addition to OAMZL, including CLL (2 cases), salivary-gland MALT lymphoma (1 case), gastric MALT lymphoma (1 case), diffuse 
large B cell lymphoma (1 case, positive for HCV) and, finally, a rheumatoid factor. All these cases shared quasi-identical VH CDR3s and, furthermore, also exhibited a "stereotyped" somatic hypermutation in VH CDR2 codon 58, leading to substitution of proline for glutamine (Figure 5B).

Only $2 \mathrm{HC}-\mathrm{MBL}$ rearrangements were clustered together with sequences from different entities except CLL that were retrieved from the IMGT/LIGM-DB sequence database. The first carried a mutated BcR IG, which was quasi-identical with a rheumatoid factor. The second clustered with the BcR IG from a salivary MALT lymphoma and the autoreactive BcR IG from a patient with primary Sjögren's syndrome. 


\section{DISCUSSION}

CLL-like MBL is generally considered to represent tout court a pre-CLL state, in analogy to what Monoclonal Gammopathy of Undetermined Significance (MGUS) is for multiple myeloma. ${ }^{26}$ Along this line, a prospective cohort study demonstrated that CLL is always preceded by CLL-like MBL. ${ }^{10}$ However, the frequency of CLL-like MBL is particularly high among the general population, reaching $12 \%$ when advanced flow cytometric techniques are used, ${ }^{5}$ thereby being at least 100 times more frequent than CLL and highlighting the fact that not all MBL carry the same risk of clinical progression. ${ }^{15}$ It is therefore of crucial relevance to clearly distinguish those cases that are more likely to progress and require special clinical attention during follow-up. Studying the biological characteristics of MBL may then help us identify prognostic markers that correlate with progression toward CLL, sparing the majority of cases from unnecessary psychological distress and continuous medical attention with the obvious social and economic implications.

So far, the absolute clonal B lymphocyte count is the only factor that was found to hold some prognostic value for the progression of $\mathrm{MBL}$ into CLL requiring treatment. ${ }^{13}$ Based on survival curves, it has been proposed that MBL may be subdivided into two groups, Low Count and High Count, by being below or above a suggested cut-off of $0.5 \times 10^{9}$ clonal cells/l, respectively. ${ }^{14,16}$ Recently, our group demonstrated that LC-MBL carries distinct biological features, including the IG gene repertoire, from CLL, both mutated and unmutated, and supported the idea that this entity may not always be equivalent to a premalignant state. ${ }^{4}$

Here, we analyzed a large, multinational cohort of CLL-like MBL cases, both LC and $\mathrm{HC}$, which allowed us to perform informative IG gene repertoire comparisons of LCMBL, HC-MBL, CLL-0 and CLL-all stages. For all comparisons, we followed the cutoff of $0.5 \times 10^{9}$ clonal cells/l to discriminate LC from HC-MBL. This cut-off was not only suggested by the literature but also an educated choice; use of other cut-off values, (namely 1.0 and $1.5 \times 10^{9} / \mathrm{l}$ ) did not affect our conclusions since our MBL cohort was polarized towards carrying either really low-count clones or $>1.5 \times 10^{9}$ clonal B cells/l, and cases with a clonal $B$ cell count between 0.5 and $1.5 \times 10^{9} / /$ were in essence very few $(n=17)$ (Figure 1).

The analysis of the IGHV genes expressed by LC-MBL revealed significant differences from HC-MBL as well as from CLL-all stages in terms of both gene usage and, perhaps more importantly, the frequency of BcR stereotypy. Interestingly, HCMBL showed great similarity with CLL, especially CLL stage Rai 0. In contrast, compared to both HC-MBL and CLL, LC-MBL exhibits pronounced differences e.g. 
suppressed frequency of the IGHV1-69 gene and overrepresentation of the IGHV4$59 / 61$ genes, confirming our previous findings. ${ }^{4}$ The underrepresentation of the IGHV1-69 gene in LC-MBL may be associated with the overall paucity of stereotyped BcRs in this category, as this gene shows a remarkable propensity to be utilized in stereotyped rearrangements in CLL. ${ }^{22,23}$

The latter have been shown to be a distinctive feature of CLL being present in almost one-third of cases. ${ }^{21,22}$ Hence, our present finding that stereotyped receptors are indeed rare in LC-MBL is particularly noteworthy, again suggesting a phylogenetic distance from CLL, whereas HC-MBL is characterized by a much higher frequency of BcR stereotypes, surprisingly similar to that of CLL stage 0 , to which again seems to be more closely related at the biological level.

One also has to consider that HC-MBL cases have been demonstrated to carry a risk of evolution into CLL needing treatment at a sizeable frequency between 1$4 \% /$ year, ${ }^{14,16,27}$ while CLL stage 0 patients will require therapy at an approximate rate of $5 \% /$ year. ${ }^{28}$ This indicates that both entities comprise a mixture of cases with distinct overall propensity of progression and suggests the need to identify molecular or clinical signatures, besides the official threshold of $5 \times 10^{9}$ clonal $B$ cells/l, to distinguish between progressive and non-progressive cases regardless the actual MBL or CLL diagnosis.

From a clinical standpoint, our immunogenetic data further supports the idea that HC-MBL should be managed as CLL not requiring treatment, i.e. with regular followups. It remains to be established in prospective studies whether it may be worth evaluating the IGHV gene usage, mutational status and/or BcR stereotypy in an effort to obtain information that could be of use in identifying the small fraction of cases that will eventually progress. In this respect, it is worth mentioning that the most common subset recognized among this $\mathrm{HC}-\mathrm{MBL}$ cohort was subset \#2 (expressing IGHV3-21/IGHJ6 VH CDR3; 10\%, 7/72 stereotyped HC-MBL clones). Subset \#2 is conspicuous for its aggressive clinical behavior, ${ }^{29-31}$ so it would not be unreasonable to speculate that a HC-MBL case also expressing this stereotype should be followed more closely, though this remains to be proven.

On the other hand, the overrepresentation of the IGHV4-59/61 genes in LC-MBL closely parallels the significant increase of this gene among elderly individuals, ${ }^{32}$ suggesting that it might simply mirror the age-related repertoire restriction occurring in healthy individuals with age..$^{33-35}$ This further supports the possibility that LC-MBL may simply represent an aspect of the physiological process of immune-senescence. Similar repertoire restrictions are well established for the $T$ cell peripheral pool, where the TCR diversity appears to collapse with age, especially in the $\mathrm{CD} 8^{+}$but also 
in the CD4 ${ }^{\text {high }} C D 8^{\text {low }}$ compartment. ${ }^{36-38}$ The frequent presence in the elderly of clonal expansions among the $\mathrm{CD}^{+} \mathrm{T}$ cells, but even more interestingly among normally negligible peripheral T-cell subsets, closely resembles CLL-like MBL in healthy individuals, the frequency of which is also age-related. $5,39,40$ The finding of "public signatures" among BcR stereotypes in LC-MBL further supports the notion that LC$M B L$ does not necessarily represent a preleukemic condition rather it may reflect repertoire restriction/polarization due to immune senescence and/or chronic antigenic stimulation. ${ }^{41}$ Along the same line lies the conspicuously increased frequency of multiple productive rearrangements in LC-MBL. Expression of multiple antigen receptors has been reported for autoreactive $B$ cells, where constant exposure to self-antigens may ultimately lead to allelic "inclusion" as an editing mechanism (otherwise described as "receptor dilution"). ${ }^{42-44}$ In sharp contrast, the frequency of multiple productive rearrangements in HC-MBL was similar to the one reported for CLL. ${ }^{45}$ Altogether, these findings support the notion that LC-MBL might constitute another example where clonality does not directly translate to malignancy and cast serious doubts on its relation with CLL.

Against this contention, the seemingly indolent nature of LC-MBL has to be reconciled with the finding of the same cytogenetic abnormalities that are frequently encountered in CLL (del13q, trisomy 12, del17p). ${ }^{15}$ However, as we recently reported, all LC-MBL cases, even those cases carrying cytogenetically abnormal clones, showed no evidence of progression from the low-count state during a median follow-up time of 34 months. ${ }^{15}$ This may well stand in analogy to the detection of "lymphoma-associated" translocations in circulating B cells of healthy individuals, e.g. the follicular lymphoma-associated $t(14 ; 18)$ (with reported frequencies ranging from $30 \%$ to $>70 \%)^{46-49}$ and the mantle cell lymphoma-associated $t(11 ; 14)$ (which is rarer, $1-7 \%) .{ }^{50,51}$ Altogether, it is reasonable to argue that genetic aberrations may occur early in the timeline of MBL, perhaps as a result of persistent proliferative stimuli by antigen(s), and relate more to the acquisition of the aberrant (or persistently activated?) phenotype rather than the malignant state itself.

One could argue that, since HC-MBL/CLL probably stems from a single cell that clonally expands as it gradually suffers malignant hits, it must "transit" through the LC-MBL range as well. We believe that in LC-MBL the clonal population that results from persistent antigenic stimulation is contained despite the fact that the proliferative stimuli lead to the acquisition of genetic aberrations. Perhaps HC-MBL/CLL may develop only when this microenvironmental drive occurs in a background of genetic predisposition, with the expanded clone having inherently different tendencies to quickly transit from the LC-MBL to the HC-MBL/CLL phase. This theory appears to 
be also supported by the polarized distribution of the MBL cases in our cohort, where few MBL cases carry a clone size of $0.5-1.5 \times 10^{9}$ cells/l, instead they carry either really low-count clones (median clonal B cell count of $0.008 \times 10^{9} /$ ) ), or clones $>1.5$ $\mathrm{x} 10^{9}$ cells $/ \mathrm{l}$.

Due to such small clonal size, LC-MBL hardly causes by itself any perturbation in the blood count differential and requires application of high-sensitivity flow cytometry protocols in order to be identified. Therefore, a physician will not likely encounter this entity in everyday practice. In any case, our immunogenetic data suggest that this condition is not clinically relevant and should not prompt monitoring neither analysis of biological prognostic markers, unless for research purposes.

In conclusion, while LC-MBL likely results from persistent antigenic stimulation, the relevant immune pathways can be distinct from those implicated in the ontogeny of CLL, especially the stereotyped category. On the contrary, HC-MBL has remarkable immunogenetic similarities to CLL-0, extending from similar IGHV gene usage to similar frequency of "CLL-specific" BcR stereotypes, which reinforce their immunobiological relatedness, besides their clinical proximity. Therefore, thorough molecular analysis and characterization of BcR stereotypy combined with long-term clinical monitoring of HC-MBL may help in clarifying the degree to which "CLLbiased" stereotypy can serve as a predictor for evolution to CLL. 


\section{ACKNOWLEDGMENTS}

This work was supported in part by the ENosAl project (code 09SYN-13-880) cofunded by the EU and the Hellenic General Secretariat for Research and Technology to KS; Cariplo Foundation (Milan, Italy) to PG and KS; Program Molecular Clinical Oncology-5 per mille number 9965 and Investigator Grant, Associazione Italiana per la Ricerca sul Cancro (AIRC - Milano, Italy) to PG. Progetti di Rilevanza Nazionale (PRIN) 2008 - Ministero Istruzione, Università e Ricerca (MIUR), Roma to PG; Progetto Finalizzato 2010 - Ministero della Salute, Roma, to PG.

\section{AUTHORSHIP}

Anna Vardi performed research, analyzed data and wrote the paper. Antonis Dagklis, Lydia Scarfò, Diane F. Jelinek, Darren J. Newton, Chi Doughty, Julia Almeida, Arancha Rodriguez-Caballero and Sallie D. Allgood performed research. Mark C. Lanasa, Agostino Cortelezzi, Ester M. Orlandi, Silvio Veronese, Marco Montillo, Andy Rawstron, Tait D. Shanafelt and Alberto Orfao provided samples and associated clinicopathological data and supervised research. Paolo Ghia and Kostas Stamatopoulos designed the study, supervised research and wrote the paper.

\section{CONFLICT-OF-INTEREST DISCLOSURE}

Andy Rawstron provides consultancy to Biogenldec. All other authors have no relevant conflicts of interest to disclose. 


\section{REFERENCES}

1. Han $\mathrm{T}$, Ozer H, Gavigan M, et al. Benign monoclonal B cell lymphocytosis--a benign variant of CLL: clinical, immunologic, phenotypic, and cytogenetic studies in 20 patients. Blood. 1984;64(1):244-252.

2. Kimby E, Mellstedt H, Bjorkholm M, Holm G. Clonal cell surface structures related to differentiation, activation and homing in B-cell chronic lymphocytic leukemia and monoclonal lymphocytosis of undetermined significance. Eur $J$ Haematol. 1989;43(5):452-459.

3. Rachel JM, Zucker ML, Fox CM, et al. Monoclonal B-cell lymphocytosis in blood donors. Br J Haematol. 2007;139(5):832-836.

4. Dagklis A, Fazi C, Sala C, et al. The immunoglobulin gene repertoire of lowcount chronic lymphocytic leukemia (CLL)-like monoclonal B lymphocytosis is different from CLL: diagnostic implications for clinical monitoring. Blood. 2009;114(1):26-32.

5. Nieto WG, Almeida J, Romero A, et al. Increased frequency (12\%) of circulating chronic lymphocytic leukemia-like B-cell clones in healthy subjects using a highly sensitive multicolor flow cytometry approach. Blood. 2009;114(1):33-37.

6. Rawstron AC, Green MJ, Kuzmicki A, et al. Monoclonal B lymphocytes with the characteristics of "indolent" chronic lymphocytic leukemia are present in $3.5 \%$ of adults with normal blood counts. Blood. 2002;100(2):635-639.

7. Ghia P, Prato G, Scielzo C, et al. Monoclonal CD5+ and CD5- B-lymphocyte expansions are frequent in the peripheral blood of the elderly. Blood. 2004;103(6):2337-2342.

8. Shanafelt TD, Ghia P, Lanasa MC, Landgren O, Rawstron AC. Monoclonal Bcell lymphocytosis (MBL): biology, natural history and clinical management. Leukemia. 2010;24(3):512-520.

9. Marti GE, Rawstron AC, Ghia P, et al. Diagnostic criteria for monoclonal Bcell lymphocytosis. Br J Haematol. 2005;130(3):325-332.

10. Landgren O, Albitar M, Ma W, et al. B-cell clones as early markers for chronic lymphocytic leukemia. N Engl J Med. 2009;360(7):659-667.

11. Goldin LR, Lanasa MC, Slager SL, et al. Common occurrence of monoclonal B-cell lymphocytosis among members of high-risk CLL families. $\mathrm{Br} J$ Haematol. 2010;151(2):152-158.

12. WHO Classification of Tumours of Haematopoietic and Lymphoid Tissue (IARC WHO Classification of Tumours); 2008. 
13. Rawstron $A C$, Shanafelt $T$, Lanasa $M C$, et al. Different biology and clinical outcome according to the absolute numbers of clonal B-cells in monoclonal B-cell lymphocytosis (MBL). Cytometry B Clin Cytom. 2010;78 Suppl 1:S19-23.

14. Rawstron AC, Bennett FL, O'Connor SJ, et al. Monoclonal B-cell lymphocytosis and chronic lymphocytic leukemia. N Engl J Med. 2008;359(6):575583.

15. Fazi C, Scarfo L, Pecciarini L, et al. General population low-count CLL-like MBL persists over time without clinical progression, although carrying the same cytogenetic abnormalities of CLL. Blood. 2011;118(25):6618-6625.

16. Rossi D, Sozzi E, Puma A, et al. The prognosis of clinical monoclonal B cell lymphocytosis differs from prognosis of Rai 0 chronic lymphocytic leukaemia and is recapitulated by biological risk factors. Br J Haematol. 2009;146(1):64-75.

17. Murray F, Darzentas N, Hadzidimitriou A, et al. Stereotyped patterns of somatic hypermutation in subsets of patients with chronic lymphocytic leukemia: implications for the role of antigen selection in leukemogenesis. Blood. 2008;111(3):1524-1533.

18. Lefranc MP, Giudicelli V, Ginestoux C, et al. IMGT, the international ImMunoGeneTics information system. Nucleic Acids Res. 2009;37:D1006-1012.

19. Brochet X, Lefranc MP, Giudicelli V. IMGT/V-QUEST: the highly customized and integrated system for IG and TR standardized V-J and V-D-J sequence analysis. Nucleic Acids Res. 2008;36:W503-508.

20. Lefranc MP, Pommie C, Ruiz M, et al. IMGT unique numbering for immunoglobulin and $\mathrm{T}$ cell receptor variable domains and $\lg$ superfamily $\mathrm{V}$-like domains. Dev Comp Immunol. 2003;27(1):55-77.

21. Agathangelidis A, Darzentas N, Hadzidimitriou A, et al. Stereotyped B-cell receptors in one-third of chronic lymphocytic leukemia: a molecular classification with implications for targeted therapies. Blood. 2012;119(19):4467-4475.

22. Darzentas N, Hadzidimitriou A, Murray F, et al. A different ontogenesis for chronic lymphocytic leukemia cases carrying stereotyped antigen receptors: molecular and computational evidence. Leukemia. 2010;24(1):125-132.

23. Stamatopoulos K, Belessi C, Moreno C, et al. Over $20 \%$ of patients with chronic lymphocytic leukemia carry stereotyped receptors: Pathogenetic implications and clinical correlations. Blood. 2007;109(1):259-270.

24. Kostareli E, Gounari M, Janus A, et al. Antigen receptor stereotypy across Bcell lymphoproliferations: the case of IGHV4-59/IGKV3-20 receptors with rheumatoid factor activity. Leukemia. 2012;26(5):1127-1131. 
25. Dagklis A, Ponzoni M, Govi S, et al. Immunoglobulin gene repertoire in ocular adnexal lymphomas: hints on the nature of the antigenic stimulation. Leukemia. 2012;26(4):814-821.

26. Kern W, Bacher U, Haferlach C, et al. Monoclonal B-cell lymphocytosis is closely related to chronic lymphocytic leukaemia and may be better classified as early-stage CLL. Br J Haematol. 2012;157(1):86-96

27. Shanafelt TD, Kay NE, Rabe KG, et al. Brief report: natural history of individuals with clinically recognized monoclonal B-cell lymphocytosis compared with patients with Rai 0 chronic lymphocytic leukemia. J Clin Oncol. 2009;27(24):39593963.

28. Scarfo L, Zibellini S, Tedeschi A, et al. Impact of B-cell count and imaging screening in CMBL: any need to revise the current guidelines? Leukemia. 2012;26(7):1703-1707.

29. Tobin $G$, Thunberg $U$, Johnson $A$, et al. Somatically mutated $\lg V(H) 3-21$ genes characterize a new subset of chronic lymphocytic leukemia. Blood. 2002;99(6):2262-2264.

30. Tobin G, Thunberg $U$, Johnson A, et al. Chronic lymphocytic leukemias utilizing the VH3-21 gene display highly restricted Vlambda2-14 gene use and homologous CDR3s: implicating recognition of a common antigen epitope. Blood. 2003;101(12):4952-4957.

31. Thorselius M, Krober A, Murray F, et al. Strikingly homologous immunoglobulin gene rearrangements and poor outcome in VH3-21-using chronic lymphocytic leukemia patients independent of geographic origin and mutational status. Blood. 2006;107(7):2889-2894.

32. Geiger KD, Klein U, Brauninger A, et al. CD5-positive B cells in healthy elderly humans are a polyclonal B cell population. Eur J Immunol. 2000;30(10):29182923.

33. Ghia P, Melchers F, Rolink AG. Age-dependent changes in B lymphocyte development in man and mouse. Exp Gerontol. 2000;35(2):159-165.

34. Klinman NR, Kline GH. The B-cell biology of aging. Immunol Rev. 1997;160:103-114.

35. LeMaoult J, Szabo P, Weksler ME. Effect of age on humoral immunity, selection of the B-cell repertoire and B-cell development. Immunol Rev. 1997;160:115-126.

36. Ghia P, Prato G, Stella S, Scielzo C, Geuna M, Caligaris-Cappio F. Agedependent accumulation of monoclonal CD4+CD8+ double positive $T$ lymphocytes in the peripheral blood of the elderly. Br J Haematol. 2007;139(5):780-790. 
37. Naylor K, Li G, Vallejo AN, et al. The influence of age on $\mathrm{T}$ cell generation and TCR diversity. J Immunol. 2005;174(11):7446-7452.

38. Wack A, Cossarizza A, Heltai S, et al. Age-related modifications of the human alphabeta $T$ cell repertoire due to different clonal expansions in the CD4+ and CD8+ subsets. Int Immunol. 1998;10(9):1281-1288.

39. Hauswirth AW, Almeida J, Nieto WG, et al. Monoclonal B-cell lymphocytosis (MBL) with normal lymphocyte counts is associated with decreased numbers of normal circulating B-cell subsets. Am J Hematol. 2012;87(7):721-724.

40. Rawstron AC. Monoclonal B-cell lymphocytosis. Hematology Am Soc Hematol Educ Program. 2009;430-439.

41. Casabonne D, Almeida J, Nieto W, et al. Common infectious agents and monoclonal B-cell lymphocytosis: a cross-sectional epidemiological study among healthy adults. Plos ONE. 2012;7(12):e52808

42. Kenny JJ, Rezanka LJ, Lustig A, et al. Autoreactive B cells escape clonal deletion by expressing multiple antigen receptors. J Immunol. 2000;164(8):41114119.

43. Liu S, Velez M, Humann J, et al. Receptor editing can lead to allelic inclusion and development of $\mathrm{B}$ cells that retain antibodies reacting with high avidity autoantigens. J Immunol. 2005;175(8):5067-5076.

44. $\quad \mathrm{Li} \mathrm{Y,} \mathrm{Li} \mathrm{H,} \mathrm{Weigert} \mathrm{M.} \mathrm{Autoreactive} \mathrm{B} \mathrm{cells} \mathrm{in} \mathrm{the} \mathrm{marginal} \mathrm{zone} \mathrm{that} \mathrm{express}$ dual receptors. J Exp Med. 2002;195(2):181-188.

45. Langerak AW, Davi F, Ghia $P$, et al. Immunoglobulin sequence analysis and prognostication in CLL: guidelines from the ERIC review board for reliable interpretation of problematic cases. Leukemia. 2011;25(6):979-984.

46. Liu Y, Hernandez AM, Shibata D, Cortopassi GA. BCL2 translocation frequency rises with age in humans. Proc Natl Acad Sci U S A. 1994;91(19):89108914.

47. Dolken G, Illerhaus G, Hirt C, Mertelsmann R. BCL-2/JH rearrangements in circulating $B$ cells of healthy blood donors and patients with nonmalignant diseases. J Clin Oncol. 1996;14(4):1333-1344.

48. Schuler F, Hirt C, Dolken G. Chromosomal translocation $t(14 ; 18)$ in healthy individuals. Semin Cancer Biol. 2003;13(3):203-209.

49. Roulland S, Navarro JM, Grenot P, et al. Follicular lymphoma-like B cells in healthy individuals: a novel intermediate step in early lymphomagenesis. J Exp Med. 2006;203(11):2425-2431. 
50. Lecluse Y, Lebailly P, Roulland S, Gac AC, Nadel B, Gauduchon P. t(11;14)positive clones can persist over a long period of time in the peripheral blood of healthy individuals. Leukemia. 2009;23(6):1190-1193.

51. Hirt C, Schuler F, Dolken L, Schmidt CA, Dolken G. Low prevalence of circulating $\mathrm{t}(11 ; 14)(\mathrm{q} 13 ; \mathrm{q} 32)$-positive cells in the peripheral blood of healthy individuals as detected by real-time quantitative PCR. Blood. 2004;104(3):904-905. 
TABLES

\begin{tabular}{|l|c|c|}
\hline & LC-MBL & HC-MBL \\
\hline MBL cell range $\left(\mathbf{x} 10^{\mathbf{9}} / \mathbf{I}\right)$ & $0-0.5$ & $0.5-5.0$ \\
\hline Number of cases & 60 & 273 \\
\hline Number of productive rearrangements & 72 & 283 \\
\hline Median B cell count $(\mathbf{x} \mathbf{1 0}$ //) & 0.14 & 3.52 \\
\hline Median MBL cell count (x10 $/ \mathbf{l})$ & 0.008 & 3.38 \\
\hline MBL/B cell percentage (average) & $21 \%$ & $93 \%$ \\
\hline Median absolute lymphocyte count $\mathbf{( x 1 0}$ /I) & 2.17 & 6.0 \\
\hline
\end{tabular}

Table 1. Characteristics of the MBL cohort regarding absolute and relative clonal size. 


\section{FIGURES}

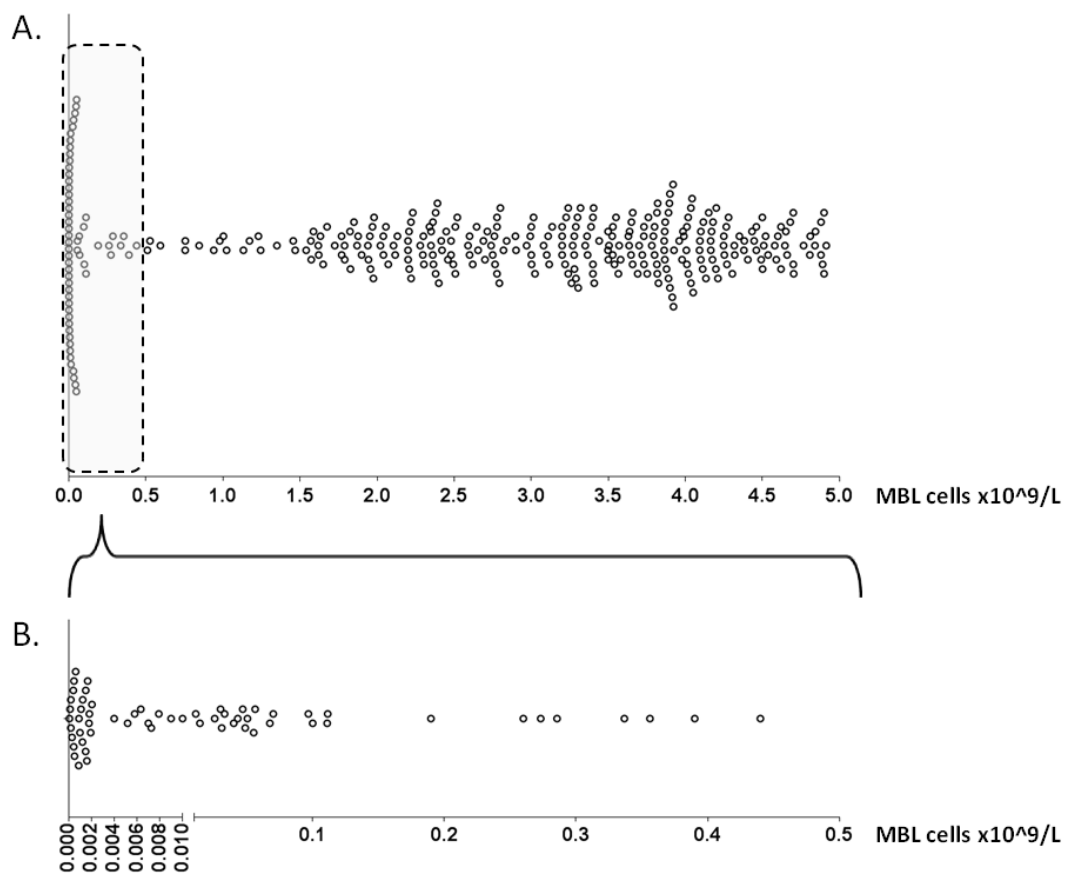

Figure 1. A) Distribution of all MBL cases according to clonal size. Each case is represented with a dot. The cohort is polarized towards carrying either really small clones or $>1.5 \times 10^{9} \mathrm{MBL}$ cells/L. B) Distribution of LC-MBL cases according to clonal size. For reasons of better visualization, the $x$ axis is split in two differently scaled segments $(<0.005$ and $\geq 0.005 \mathrm{MBL}$ cells/L). Most LC-MBL cases carry clones consisting of $<0.1 \times 10^{9} \mathrm{MBL}$ cells $/ \mathrm{L}$. 


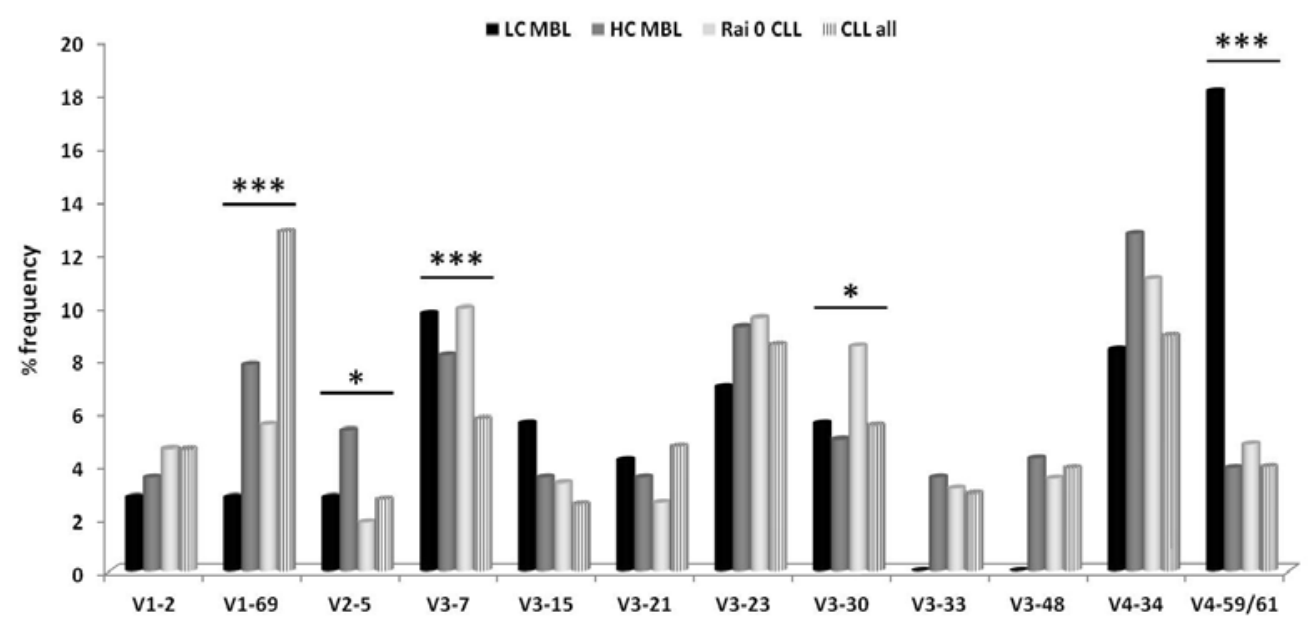

Figure 2. IGHV gene repertoire comparison in low count $M B L$ versus high count MBL versus CLL-0 versus CLL-all stages.

Differences at level of statistical significance $p<0.05$ and $p<0.001$ are marked with [*] and [**], respectively. 
From bloodjournal.hematologylibrary.org at BIBLIOTECA POLO SAN PAOLO on April 22, 2013. For personal use only.

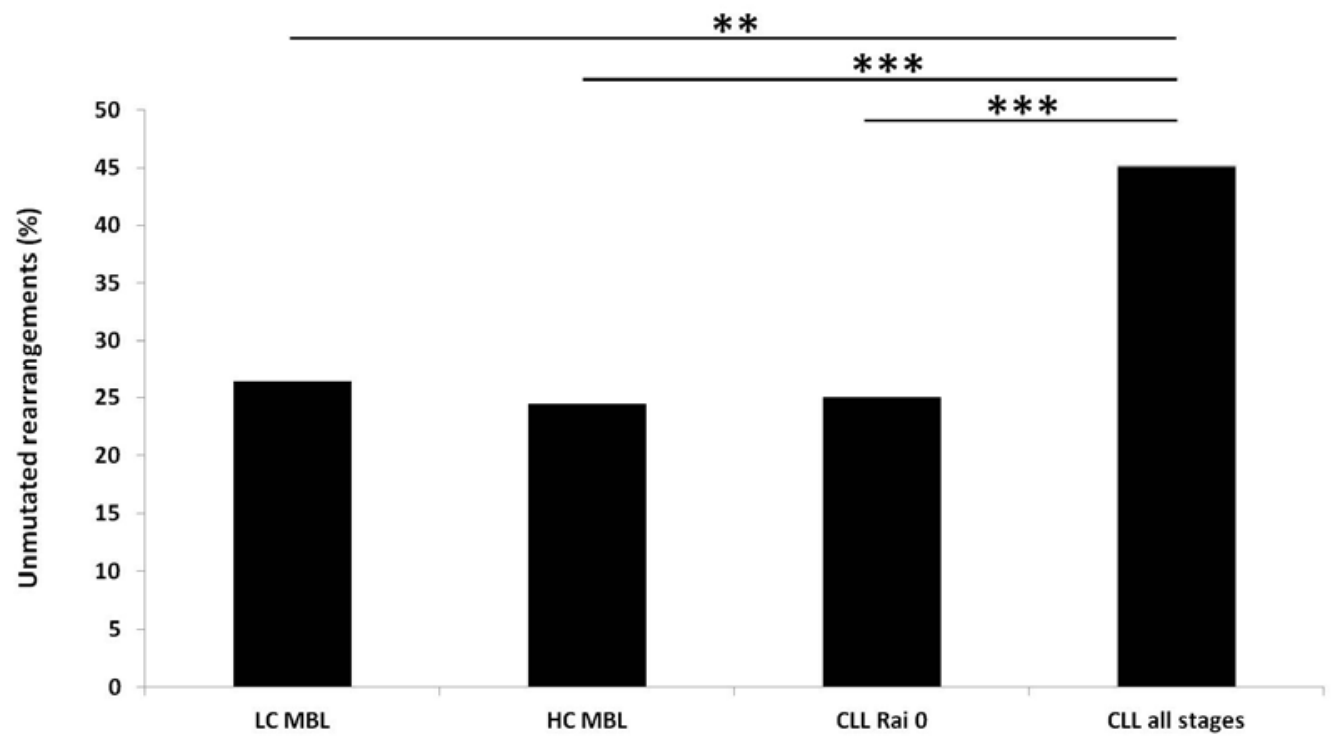

Figure 3. The frequency (\%) of unmutated IG rearrangements ( $\geq 98 \%$ germline identity) in MBL and CLL.

Differences at level of statistical significance $p<0.01$ and $p<0.001$ are marked with $\left[{ }^{* \star}\right]$ and $[* \star]$, respectively. 
From bloodjournal.hematologylibrary.org at BIBLIOTECA POLO SAN PAOLO on April 22, 2013. For personal use only.

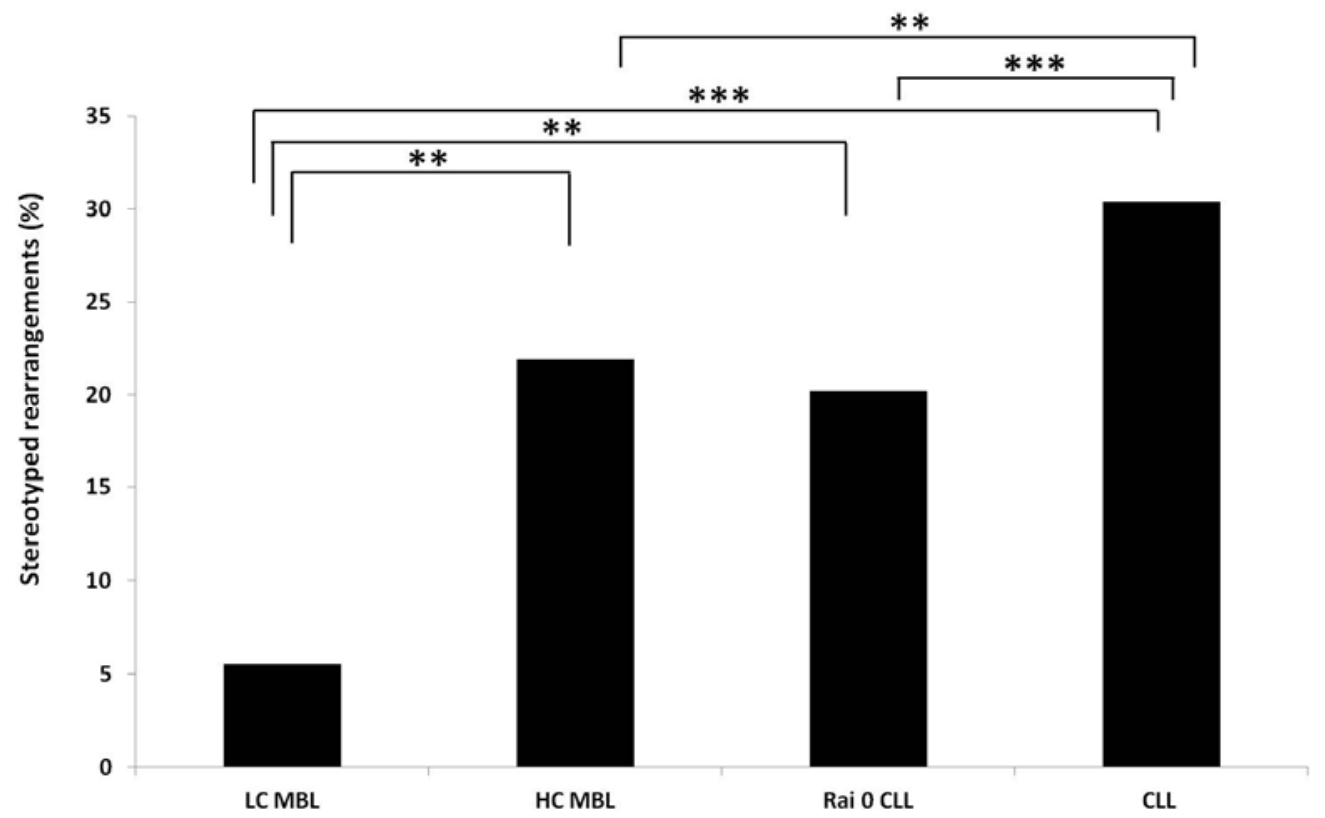

Figure 4. The frequency (\%) of BcR IG stereotypy in MBL and CLL.

Differences at level of statistical significance $p<0.01$ and $p<0.001$ are marked with $\left[{ }^{\star *}\right]$ and $\left[{ }^{\star \star *}\right.$, respectively. 
A

\begin{tabular}{|c|c|c|}
\hline 87VB & MBL, LC & ARGDYYDSSGHYHDAFDI \\
\hline 9577 & OAMZL & $\ldots y$ \\
\hline AF216825 & Salivary gland MALT lymphoma & $\cdots$ Y Y F $\cdots$ C \\
\hline AF303909 & DLBCL, HCV-related & $\cdots$. . Y Y F S $\ldots$ \\
\hline AY281326 & Gastric MALT lymphoma & $\cdots$. $\cdots$ SFI $\ldots$ \\
\hline NL-01-0121 & CLL & $\cdots F-E \cdots S-N \cdots V$ \\
\hline NL-01-0591 & CLL & $\cdots F-T$ T - Y F N - . \\
\hline U85242 & Rheumatoid factor & $\ldots \mathrm{N} \ldots \ldots$ \\
\hline
\end{tabular}

B

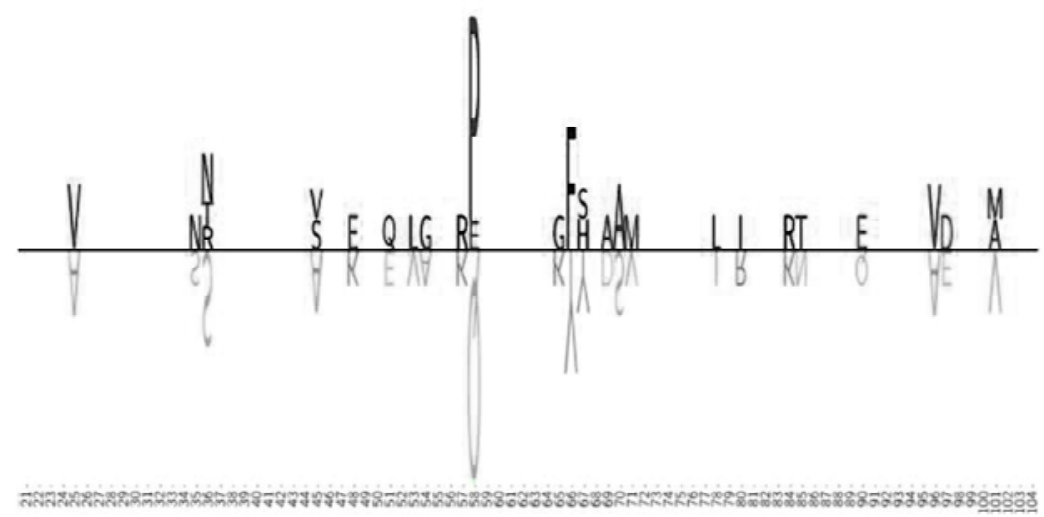

Figure 5. A) VH CDR3 similarity between the LC-MBL case $87 \mathrm{VB}$ and unrelated sequences. Dashes indicate identities. B) Somatic hypermutation in the $\mathrm{VH}$ region of the LC-MBL case 87VB and co-clustered sequences, as visualized using TeXshade peptide alignment tool (http://workbench.sdsc.edu/). The VH region is shown from IMGT positions 21 to 104. The letters above the line represent the amino acid changes, whereas the letters shown upside-down below the line represent the corresponding germ line amino acids of the IGHV gene. The size of the amino acid symbol represents the relative frequency of that amino acid at that position relative to all other mutations at that position in the certain IGHV group of sequences. Blank spaces represent amino acids that are unchanged in comparison to the germ line sequence. 